\title{
Metabolic landscape of advanced gastric cancer according to HER2 and its prognostic implications
}

\author{
Chan-Young Ock ${ }^{1}$ Tae-Yong Kim ${ }^{1,2} \cdot$ Kyung-Hun Lee ${ }^{1,2} \cdot$ Sae-Won Han $^{1,2}$. \\ Seock-Ah Im ${ }^{1,2}$ - Tae-You Kim ${ }^{1,2} \cdot$ Yung-Jue Bang ${ }^{1,2} \cdot$ Do-Youn Oh ${ }^{1,2}$
}

Received: 18 December 2014/ Accepted: 29 April 2015/Published online: 23 May 2015

(c) The International Gastric Cancer Association and The Japanese Gastric Cancer Association 2015

\begin{abstract}
Background In advanced gastric cancer (AGC), HER2 is a validated therapeutic target. However, the metabolic landscape of AGC based on HER2 status has not been reported. Furthermore, the prognostic value of HER2 in AGC is under debate. The purpose of this study was to determine the metabolic landscape and prognosis on the basis of HER2 status in AGC.

Methods We analyzed 866 AGC patients treated with palliative chemotherapy and whose HER2 status was evaluated. HER2 positivity was defined as HER2 IHC $3+$ or $H E R 2 / C E P 17$ ratio $\geq 2$. Among them, 363 patients were evaluated with ${ }^{18} \mathrm{~F}$ FDG-PET before chemotherapy. We analyzed mSUV (maximal standardized uptake value) according to HER2 status and clinical outcomes.

Results Among 866 patients, 225 (26.0\%) had HER2+ GC. The mSUV of HER2+ GC was significantly higher than that of HER2- GC (12.6 vs. 8.7, $p<0.001)$. Increased HER 2 IHC positivity was correlated with increased mSUV (IHC-: 8.1, IHC 1+: 8.2, 2+: 11.4, 3+: 13.2, $p<0.001)$. Excluding HER2+ patients who received HER2-targeting agents, OS of patients was not different by HER2 status (12.5 vs. 11.9 months, $p=0.688)$. However, according to tumor metabolism, patients with higher
\end{abstract}

Electronic supplementary material The online version of this article (doi:10.1007/s10120-015-0504-1) contains supplementary material, which is available to authorized users.

Do-Youn Oh

ohdoyoun@snu.ac.kr

1 Department of Internal Medicine, Seoul National University Hospital, 101 Daehak-ro, Jongno-gu, Seoul 110-744, Korea

2 Cancer Research Institute, Seoul National University College of Medicine, Seoul, Korea
mSUV showed worse OS regardless of HER2 positivity (mSUV $<12.8: 14.8, \geq 12.8: 8.6$ months, $p<0.001$ ).

Conclusion Tumor metabolism of AGC adversely influenced OS under treatment with cytotoxic chemotherapy. Tumor metabolism was higher in HER2+ AGC than HER2-. However, HER2 was not a prognostic factor in patients who received chemotherapy without HER2-targeting agents.

Keywords Gastric cancer - HER2 - c-erbB2 - Prognosis · Metabolism

\section{Introduction}

Gastric cancer (GC) is the second leading cause of cancer deaths worldwide. Although there has been progress in the development of cytotoxic chemotherapy, overall survival (OS) of advanced gastric cancer (AGC) patients remains at 10-12 months [1]. Recently, the benefit of second-line chemotherapy has been proven by phase III trials [2,3].

Gastric cancer is not a single disease. Deng et al. [4] performed a comprehensive genomic analysis of gastric cancer and identified 22 recurrent genetic alterations in gastric cancer. Interestingly enough, they found that five distinct gastric cancer subgroups could be defined by specific alterations, that is, amplifications of $H E R 2, F G F R$, $K R A S, E G F R$, and MET. Dulak et al. [5] also reported similar results in somatic copy number aberration analysis using high-density genomic profiling arrays in gastric/esophageal tumors. These genes can be targeted for the development of new targeted agents. Among these molecular targets, the first successfully validated one was HER2. Efficacy of an anti-HER2 treatment was demonstrated by the ToGA trial [6]. In contrast to breast cancer in 
which HER2 is known as a poor prognostic factor [7], the prognostic value of HER2 in gastric cancer is still controversial. Even though some studies have shown that HER2-positivity in gastric cancer is associated with poor outcomes and less sensitive to cytotoxic chemotherapy [811], others have demonstrated that HER2 expression does not influence overall prognosis in gastric cancer [12-15]. Moreover, it has been getting harder to determine the prognostic value of HER2 per se in AGC after routine prescription of trastuzumab for HER2-positive patients, which can significantly prolong OS [6].

Even though several subtypes of gastric cancer can be classified according to the molecular characteristics [4, 5], the metabolic activities of each subtype including HER2 have not been determined so far. Fluorine-18 $\left({ }^{18} \mathrm{~F}\right)$ fluorodeoxyglucose positron emission tomography (FDGPET) is a functional imaging method for the measurement of tumor glucose utilization, using a radioactive tracer bound to FDG, a glucose analog.

The purpose of this study was to evaluate the metabolic landscape of gastric cancer using ${ }^{18} \mathrm{~F}$ FDG-PET considering HER2 and to determine the prognostic value of HER2 in advanced gastric cancer.

\section{Materials and methods}

\section{Patients}

This study is a retrospective analysis of de-identified patientlevel data from collected medical charts. The study was designed to compare the metabolic features and prognostic value of HER2 in AGC. Patients who received palliative cytotoxic chemotherapy for AGC and whose HER2 status was evaluated at Seoul National University Hospital, Republic of Korea, from 2004 to 2013 were included.

The overall survival (OS) was estimated from the date of diagnosis of inoperable locally advanced, metastatic, or recurrent gastric cancer to the date of death or last known follow-up date. HER2 positivity was defined as HER2 IHC $3+$ or FISH-positive [HER2/CEP17 (centromere enumerator probe 17) ratio $\geq 2$ ], according to the ToGA study [6]. We used PathVysion HER-2 DNA probe kit (Vysis) for assessing the HER2/CEP17 ratio and HER2 gene copy number (GCN). All computerized tomography (CT) scans were reviewed. According to the standard protocol of our hospital, FDG was injected after fasting for $8 \mathrm{~h}$, and PET scans were then started $1 \mathrm{~h}$ after injection, using dedicated PET scanners (Gemini, Philips; Biograph 40, Siemens; or $\mathrm{mCT}$, Siemens). PET images were reconstructed using an iterative algorithm (ordered-subset expectation maximization). Standardized uptake value (SUV) was calculated as tissue concentration of radioactivity $(\mathrm{kBq} / \mathrm{ml})$ divided by injected dose per weight $(\mathrm{kBq} / \mathrm{g})$. To measure the maximal SUV (mSUV) of lesion, a volume of interest was placed on PET/CT fusion images to cover the whole tumor volume, and mSUV was automatically measured using an analysis software package (Syngo.via, Siemens) [16]. Tumor response was evaluated with RECIST 1.1 [17].

\section{Statistical analysis}

Pearson's chi-square test was performed to analyze the categorical variables including clinical characteristics. Analysis of variance (ANOVA) or $t$ test was applied to continuous parameters. Kaplan-Meier estimates with a logrank test of OS were done. Subgroup analyses were also performed using a Cox regression model, summarizing the hazard ratio (HR) and $95 \%$ confidence interval (CI) of each group. The optimal cutoff of mSUV discriminating between a positive or negative result, in terms of response to treatment and OS, was determined using receiver-operating characteristic (ROC) curve analyses. The cutoff of mSUV that maximized the sum of sensitivity and specificity was determined [18]. All reported $p$ values were twosided. Analyses were done with STATA version 12 (StataCorp LP; College Station, TX, USA).

\section{Ethics}

The study protocol was reviewed and approved by the Institutional Review Board of Seoul National University Hospital (H-1306-007-493). All studies were conducted according to guidelines (Declaration of Helsinki) for biomedical research.

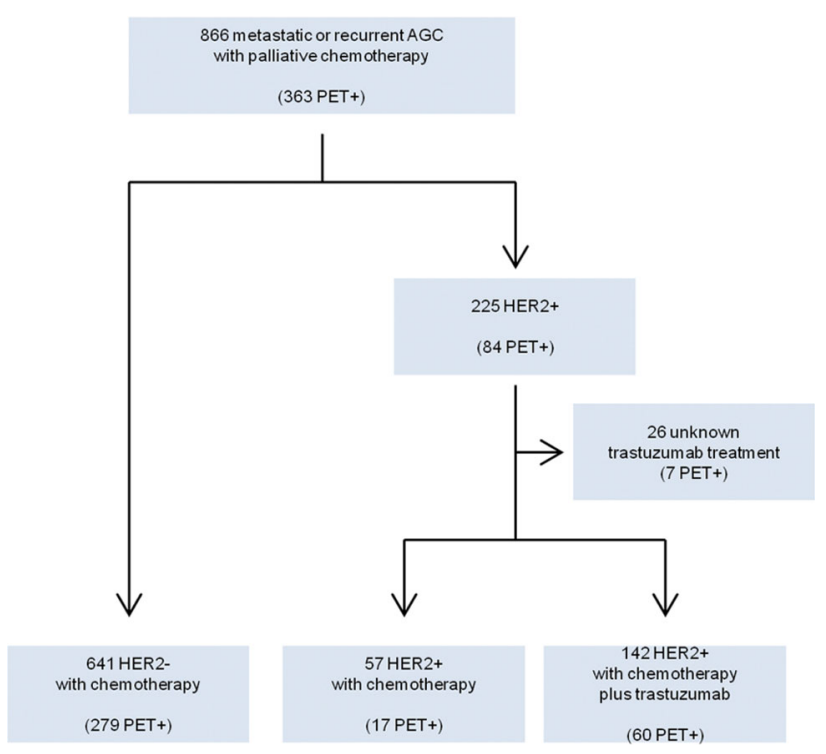

Fig. 1 Flow chart of patients in the study. $A G C$ advanced gastric cancer, PET positron emission tomography, HER2 human epidermal growth factor receptor 2 
Table 1 Patient characteristics

\begin{tabular}{llllll} 
Total & HER2 - All & HER2+ All & $p$ value & HER2 + Tmab - & $p$ value \\
$N=866$ & [A] $N=641$ & [B] $N=225$ & [A] vs. [B] & [C] $N=57$ & [A] vs. [C] \\
\hline
\end{tabular}

Age

Median (range)

$<60$ years old, $n(\%)$

$\geq 60$ years old, $n(\%)$

Sex

Male, $n(\%)$

Female, $n(\%)$

ECOG

$0-1, n(\%)$

$2-3, n(\%)$

Palliative setting

Initially metastatic, $n(\%)$

Recurrent, $n(\%)$

Tumor location

GEJ, $n(\%)$

Other stomach, $n(\%)$

Pathology type

Adenocarcinoma, $n(\%)$

Signet ring cell, $n(\%)$

Other types, $n(\%)$

Contains signet ring cell feature, $n(\%)$

Lauren

Intestinal, $n(\%)$

Mixed, n (\%)

Baseline PET

Total, $n(\%)$

mSUV

Mean \pm SE

HER2 IHC

Negative, $n(\%)$

$1+, n(\%)$

$2+, n(\%)$

$3+, n(\%)$

Total, $n(\%)$

HER2/CEP17 ratio

Median $(95 \% \mathrm{CI})$

Total, $n(\%)$

HER2 GCN

Median (95 \%CI)

Total, $n(\%)$
Diffuse, $n(\%)$

\begin{tabular}{|c|c|c|c|c|c|}
\hline $60(20-89)$ & $59(20-89)$ & $63(22-85)$ & 0.003 & $63(37-77)$ & 0.084 \\
\hline $403(46.5)$ & $314(49.0)$ & 89 (39.6) & & 22 (38.6) & \\
\hline $463(53.5)$ & $327(51.0)$ & $136(60.4)$ & 0.015 & $35(61.4)$ & 0.132 \\
\hline $620(71.6)$ & 435 (67.9) & $185(82.2)$ & & $47(82.5)$ & \\
\hline $246(28.4)$ & 206 & $40(17.8)$ & $<0.001$ & $10(17.5)$ & 0.022 \\
\hline $705(90.5)$ & $538(91.3)$ & $167(87.9)$ & & $41(83.7)$ & \\
\hline $74(9.5)$ & $51(8.7)$ & $23(12.1)$ & 0.159 & $8(16.3)$ & 0.075 \\
\hline $608(70.2)$ & $444(69.3)$ & $164(72.9)$ & & $40(70.2)$ & \\
\hline $258(29.8)$ & $197(30.7)$ & $61(27.1)$ & 0.307 & $17(29.8)$ & 0.887 \\
\hline $75(8.7)$ & $53(8.3)$ & $22(9.8)$ & & $6(10.5)$ & \\
\hline 792 (91.3) & 588 (91.7) & $203(90.2)$ & 0.489 & $51(89.5)$ & 0.557 \\
\hline $699(80.7)$ & $494(77.1)$ & $205(91.1)$ & & $55(96.5)$ & \\
\hline $152(17.6)$ & $136(21.2)$ & $16(7.1)$ & & $2(3.5)$ & \\
\hline $15(1.7)$ & $11(1.7)$ & $4(1.8)$ & $<0.001$ & $0(0)$ & 0.003 \\
\hline $222(25.6)$ & $193(30.1)$ & 29 (12.9) & $<0.001$ & $5(8.8)$ & 0.001 \\
\hline $108(36.7)$ & $66(28.3)$ & $42(68.9)$ & & $14(82.4)$ & \\
\hline $149(50.7)$ & $138(59.2)$ & $11(18.0)$ & & $2(11.8)$ & \\
\hline 37 (12.6) & $29(12.5)$ & $8(13.1)$ & $<0.001$ & $1(5.9)$ & $<0.001$ \\
\hline $363(41.9)$ & $279(43.5)$ & $84(37.3)$ & 0.105 & $17(29.8)$ & 0.045 \\
\hline $9.6 \pm 0.4$ & $8.7 \pm 0.4$ & $12.6 \pm 0.9$ & $<0.001$ & $9.1 \pm 1.3$ & 0.809 \\
\hline $276(34.5)$ & $274(42.8)$ & $2(1.0)$ & & $0(0)$ & \\
\hline $191(23.9)$ & $182(28.4)$ & $9(4.3)$ & & $3(5.7)$ & \\
\hline $184(23.0)$ & $136(21.2)$ & $48(23.0)$ & & $13(24.5)$ & \\
\hline $150(18.7)$ & $0(0)$ & $150(66.7)$ & $<0.001$ & $37(69.8)$ & $<0.001$ \\
\hline $801(92.5)$ & $592(92.4)$ & 209 (92.9) & 0.794 & $53(93.0)$ & 0.864 \\
\hline $1.2(0.9-10.8)$ & $1.1(0.8-1.6)$ & $5.8(1.2-13)$ & $<0.001$ & $4.6(1.2-11.6)$ & $<0.001$ \\
\hline $321(37.1)$ & 205 (31.9) & $116(51.6)$ & $<0.001$ & $22(38.6)$ & 0.307 \\
\hline $2.9(1.7-20.7)$ & $2.2(1.6-4.1)$ & $10(2.4-30)$ & $<0.001$ & $11.1(1.9-49.7)$ & $<0.001$ \\
\hline $293(33.8)$ & $194(30.3)$ & $99(44.0)$ & $<0.001$ & $18(31.6)$ & 0.836 \\
\hline
\end{tabular}

Bold values indicate statistically significant correlations with $p$ values less than 0.05

$C E P 17$ centromere enumerator probe 17, $C I$ confidence interval, $G C N$ gene copy number, $G E J$ gastroesophageal junction, $E C O G$ Eastern Cooperative Oncology Group performance status, HER2 human epidermal growth factor receptor 2, IHC immunohistochemistry, PET positron emission tomography, Tmab trastuzumab, $m S U V$ maximal standardized uptake value, $S E$ standardized error 


\section{Results}

\section{Patient characteristics}

A total of 866 AGC patients were analyzed (Fig. 1). The patient characteristics according to HER2 status are shown in Table 1. Among all patients, 225 (26.0\%) were HER2 positive. HER2 positivity was observed more frequently with advanced age and in males as well as non-signet-ring cell and intestinal-type pathology. Among all patients, 363 (279 HER2-, 84 HER2+) were evaluated with FDG-PET before palliative chemotherapy, and it was evenly distributed by HER2-status (Table 1). Of all cases, HER2 FISH was done in 321 cases (205 HER2-, 116 HER2+). HER2/CEP17 ratio and HER2 GCN according to HER2 IHC are summarized in Supplementary Table 1.

Among 225 HER2+ patients, 142 were treated with trastuzumab and cytotoxic chemotherapy combination, and 57 were not exposed to any HER2-targeting agents. Most commonly used first-line chemotherapy was fluoropyrimidine (5-FU, capecitabine, or TS-1) and platinum (cisplatin or oxaliplatin) combination.

\section{Metabolic landscape and metastatic pattern based on HER2 status in AGC}

The mSUV of HER2+ GC was significantly higher than that of HER2- GC (HER2-: 8.7, HER2+: 12.6, $p<0.001$, Fig. 2a), although differences in mSUV were not evident, excluding patients who were exposed to trastuzumab (HER2-: 8.7, HER2+ without trastuzumab: 9.1, $p=0.809$, Table 1; Supplementary Fig. 1).

Increased HER2 IHC positivity was correlated with increased mSUV (IHC-: 8.1, IHC 1+: 8.2, 2+: 11.4, 3+: 13.2, $p<0.001$, Fig. 2b), but a high HER2/CEP17 ratio (HCR) as well as high HER2 GCN was not correlated with increased mSUV $\quad(\mathrm{HCR}<2: 10.1, \quad \mathrm{HCR} \geq 2: 10.6$, $p=0.660, \mathrm{GCN}<6: 10.7, \quad \mathrm{GCN} \geq 6: 10.7, \quad p=0.965$, Fig. 2c, d).

Figure 3 shows the spatial distribution of tumor metabolism according to HER2 status. The SUVs of stomach
Fig. 2 Metabolic activity based on HER2 status. Maximal standardized uptake value (mSUV) according to HER2 status (a), HER2 immunohistochemistry (IHC) status (b), HER2/CEP17 ratio (c), and HER2 gene copy number $(\mathbf{d})$. Mean \pm standard error
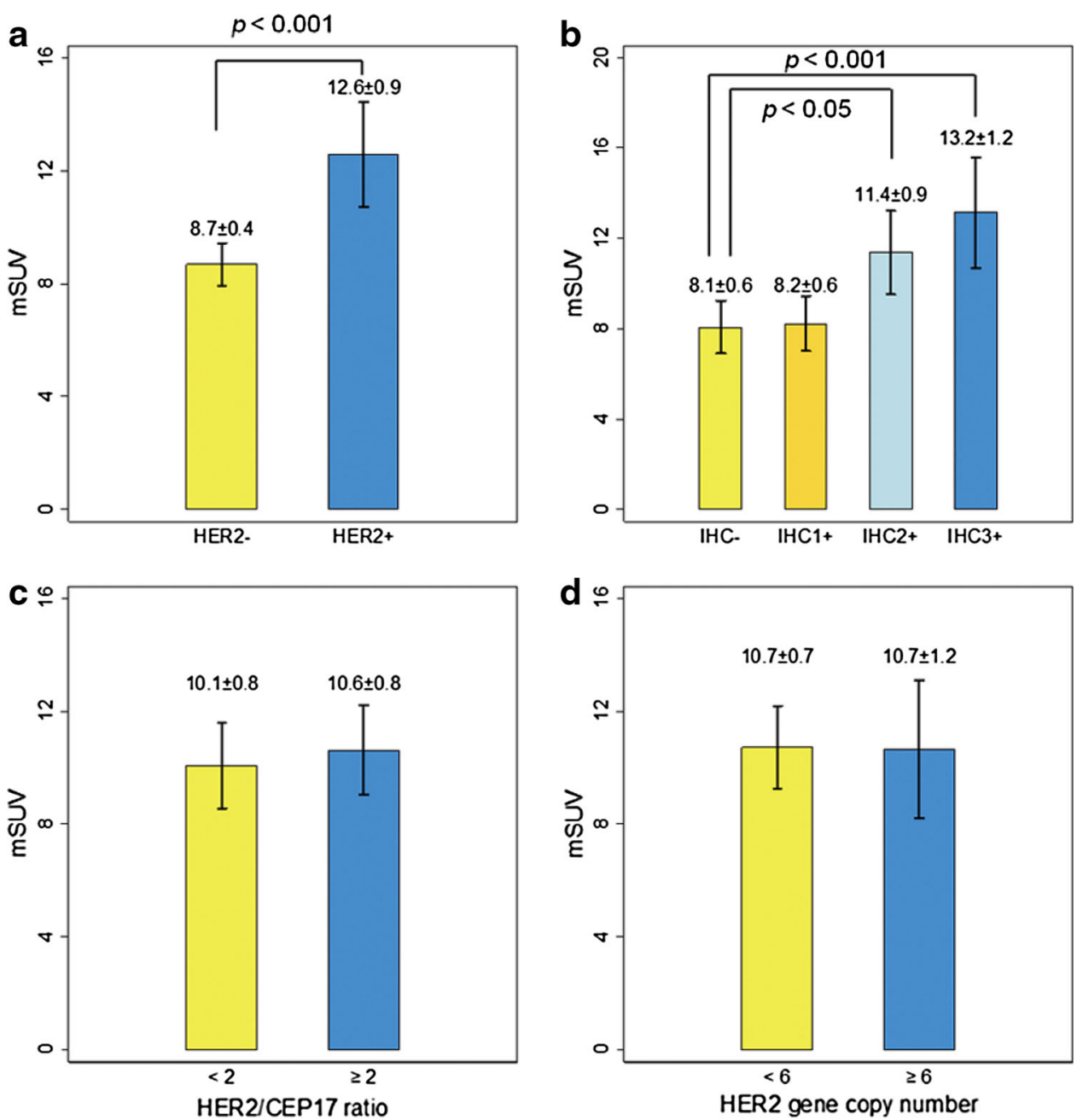

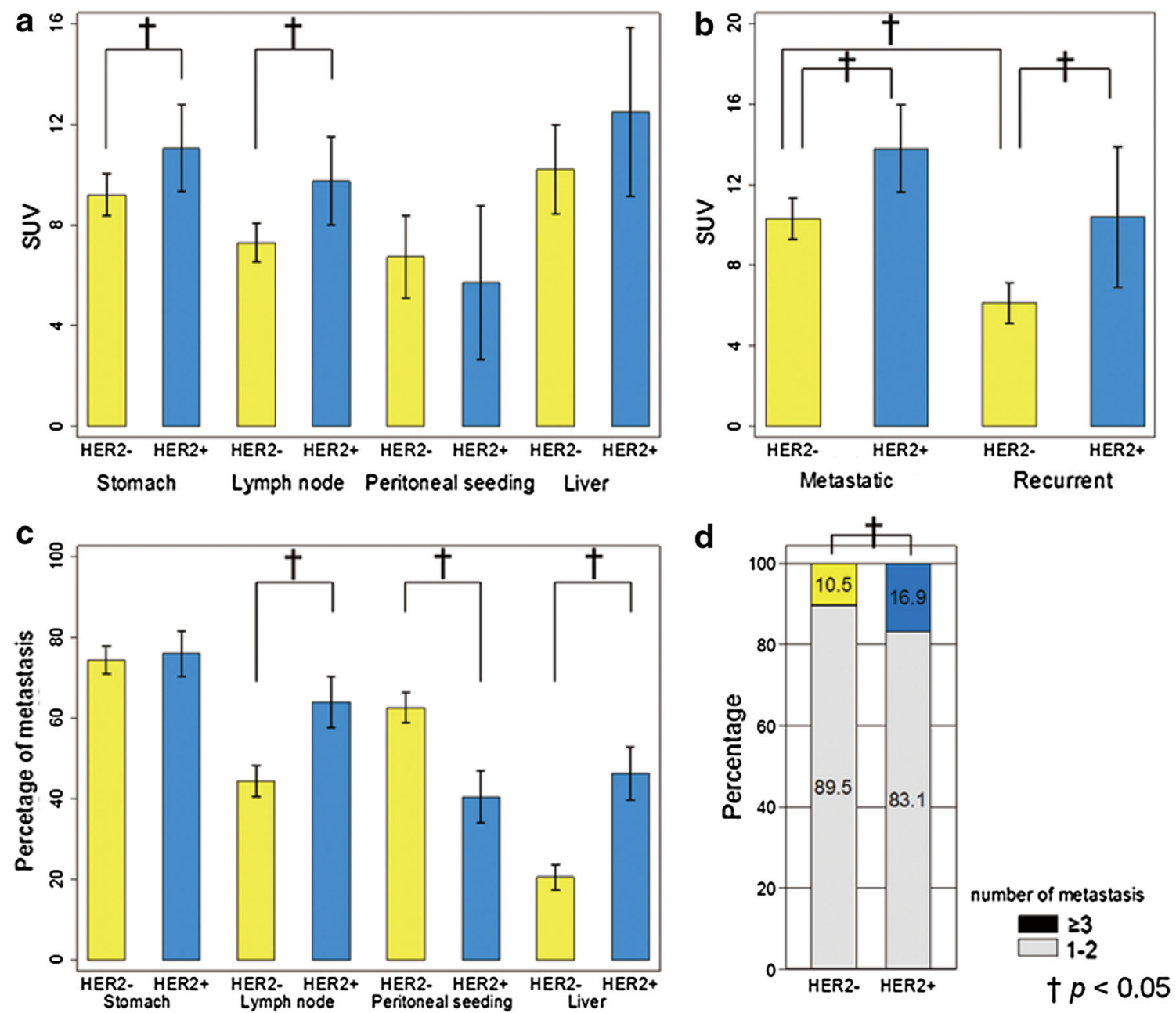

Fig. 3 Metabolic activity of metastatic lesions based on HER2 status. Standardized uptake value (SUV) of stomach, lymph node, peritoneal seeding, and liver according to HER2 status (a), SUV of metastatic and recurrent setting according to HER2 status (b). Percentage of

and lymph nodes were significantly higher in HER2+ AGC than HER2- AGC (mean stomach SUV, HER2-: 9.2, HER2+: 11.0, $p=0.042$, mean lymph node SUV, HER2-: 7.3, HER2+: 9.7, $p=0.003$, Fig. 3a). SUV of peritoneal seeding lesions was slightly higher in HER2AGC, but that of liver was higher in HER2+ AGC, although those trends were not statistically significant (mean peritoneal seeding SUV, HER2-: 6.7, HER2+: 5.7, $p=0.681$, mean liver SUV, HER2-: 10.2, HER2+: 12.5, $p=0.224$ ).

We further analyzed the SUV of lesions in initially metastatic cases compared with those in recurrent cases. In HER2 - AGC, the mSUV of initially metastatic cases was significantly higher than that of recurrent cases, and this trend was similarly observed in HER2+ AGC but failed to achieve statistical significance (HER2-, metastatic: 10.3, recurrent $6.1, p<0.001$, HER2 + , metastatic: 13.8 , recurrent: $10.4, p=0.08$, Fig. $3 b$ ). Incidences of initial recurred stomach or metastasis to lymph node, peritoneal seeding, and liver were compared according to HER2 status (c). Percentages of multiple metastases, more than 3 lesions, were compared according to HER2 status (d)

metastasis by organ are summarized in Fig. 3c. Incidences of lymph node and liver metastasis were higher in HER2+ AGC (lymph node, HER2-: $44.5 \%$, HER2+: $64.0 \%$, $p<0.001$, liver, HER2-: $20.6 \%$, HER $2+46.2 \%$, $p<0.001$ ), but peritoneal seeding was more frequently observed in HER2- AGC (HER2-: $62.6 \%$, HER2+: $40.4 \%, p<0.001)$. The number of metastatic lesions was different according to HER 2 status. The portion of multiple metastases (more than three organs) was higher in HER2+ AGC (HER2-: $10.5 \%$, HER2+: $16.9 \%, p=0.011$, Fig. 3d).

\section{Prognostic value of HER2}

The median follow-up duration of all cases was 36.7 months (95\% CI 14.7-90.2). Since trastuzumab definitely influenced survival in HER2+ AGC, we excluded HER2+ AGC patients treated with trastuzumab in 

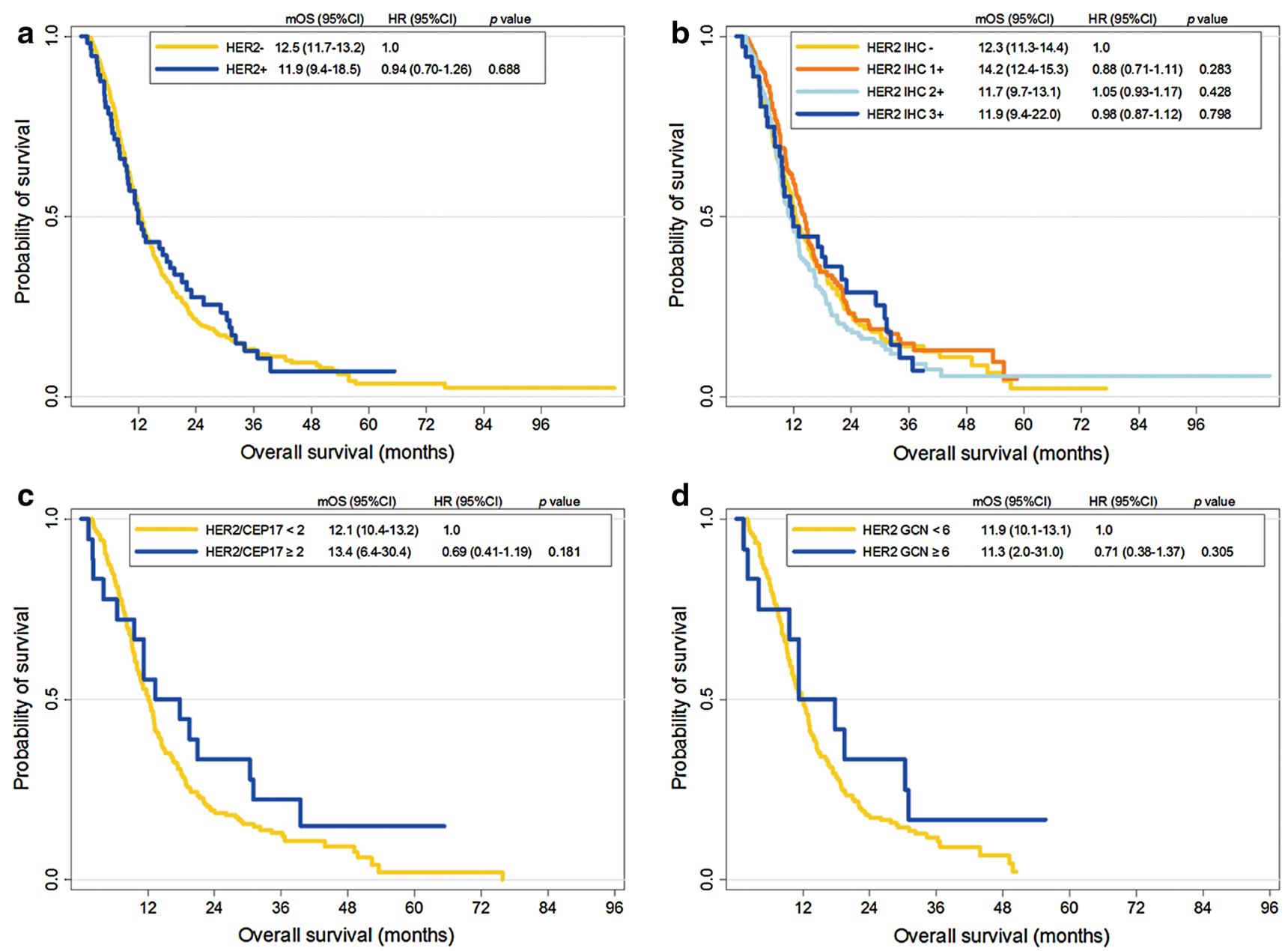

Fig. 4 Prognostic value of HER2. Kaplan-Meier curves for OS according to HER2 status (a), HER2 immunohistochemisty (IHC) status (b), HER2/CEP17 (c), and HER2 gene copy number (GCN) (d)

the following analysis. Excluding trastuzumab exposure, median overall survival of patients with intestinal-type Lauren classification was 19.1 months compared to 13.4 months in those with diffuse type (HR 1.58, $p=0.007$ ) and 13.1 months in mixed type (HR 1.73, $p=0.035$ ), respectively (Supplementary Fig. 2). However, HER2 itself did not significantly alter the prognosis (OS, HER2-: 12.5, HER2+: 11.9 months, $p=0.688$, Fig. 4a). In parallel, neither increased HER2 IHC positivity (IHC-: 12.3, IHC 1+: 14.2, 2+: 11.7, 3+: 11.9 months, $p=0.386$, Fig. 4b), nor HER2/CEP17 ratio $(\mathrm{HCR}<2$ : 12.1, HCR $\geq 2$ : 13.4 months, $p=0.181$, Fig. 4c), nor HER2 gene copy number $(\mathrm{GCN}<6$ : $11.9, \quad$ GCN $\geq 6$ : 11.3 months, $p=0.305$, Fig. 4d) influenced OS.

\section{Prognostic values of $\mathrm{mSUV}$}

ROC analysis were used to determine the optimal cutoff value of mSUV that discriminated patients with longer survival and those who respond more to first-line chemotherapy, excluding patients with trastuzumab exposure (Fig. 5a, b). ROC analysis showed that SUVs of 12.8 and 8.3 were optimal cutoff values for estimating shorter OS (less than 12 months) and response [objective response rate $(\mathrm{ORR})]$ to first-line chemotherapy, respectively. $\mathrm{Pa}$ tients with higher mSUVs of more than 8.3 showed higher ORR $\quad(\mathrm{mSUV}<8.3: 24.7 \%, \quad \mathrm{mSUV} \geq 8.3: 41.0 \%$, $p=0.004)$. However, when divided by an SUV of 12.8, patients with higher mSUV showed worse OS (mSUV < 12.8:14.8, mSUV $\geq 12.8: 8.6$ months, $p<0.001$, Fig. 5c). This trend was in concordance irrespective of HER2 status (HER2 - $\quad$ mSUV $<12.8: 14.5, \quad$ HER2 $-\quad$ mSUV $\geq$ 12.8:8.6 months, $p<0.001$, HER $2+$ mSUV $<12.8: 36 . \overline{8}$, HER2 + mSUV $\geq 12.8: 9.4$ months, $p=0.006$, Fig. 5d). Clinicopathologic characteristics of patients grouped by mSUV of 12.8 are shown in Supplementary Table 2. The group of high metabolism $(\mathrm{mSUV} \geq 12.8)$ had more initially metastatic cases and less signet ring feature pathology. Supplementary Table 3 shows the univariate and multivariate Cox analysis of OS, which indicated that 


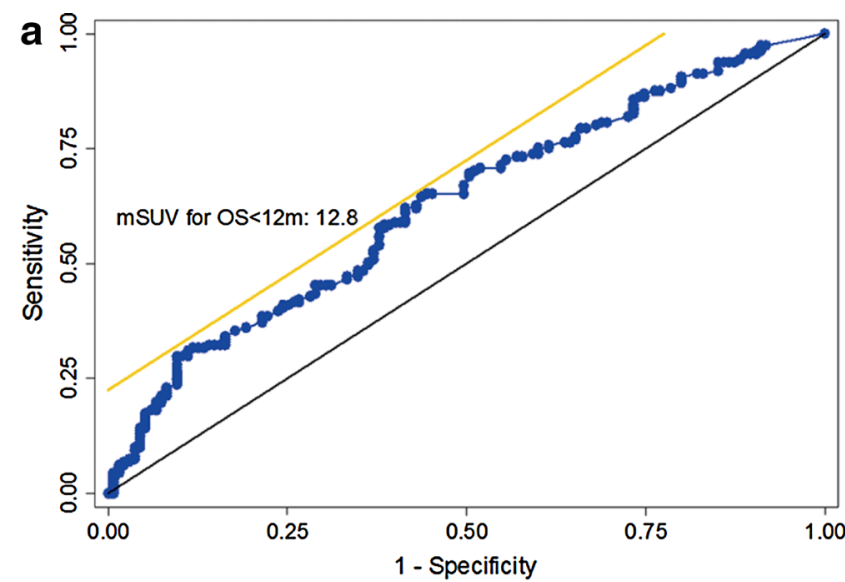

Area under ROC curve $=0.6281$

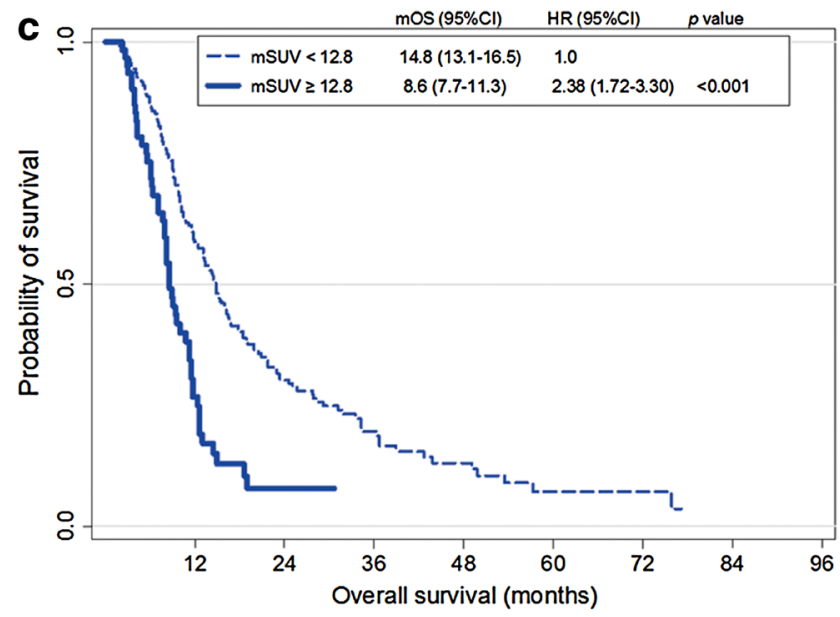

Fig. 5 Prognostic value of metabolic activity assessed by positron emission tomography. Optimal cutoff values of maximal standardized uptake value (mSUV) predicting OS shorter than 12 months (a) and

mSUV was an independent poor prognostic factor along with poor performance, peritoneal seeding, and liver metastasis (Table 2).

\section{Discussion}

In this study, we found that tumor metabolism, measured by the SUV of ${ }^{18} \mathrm{~F}$ FDG-PET, was increased in HER2+ AGC compared with HER2- AGC. HER2 itself did not impact the overall prognosis under cytotoxic chemotherapy without HER2-targeting agents; however, hypermetabolism of tumors was a poor prognostic factor irrespective of HER2 status.

The prognostic value of HER2 in AGC has been variously reported and controversial. Although some studies have reported that HER2 positivity is a poor prognostic factor in gastric cancer [8-11], others have found that HER2 has no influence on prognosis [12-15].
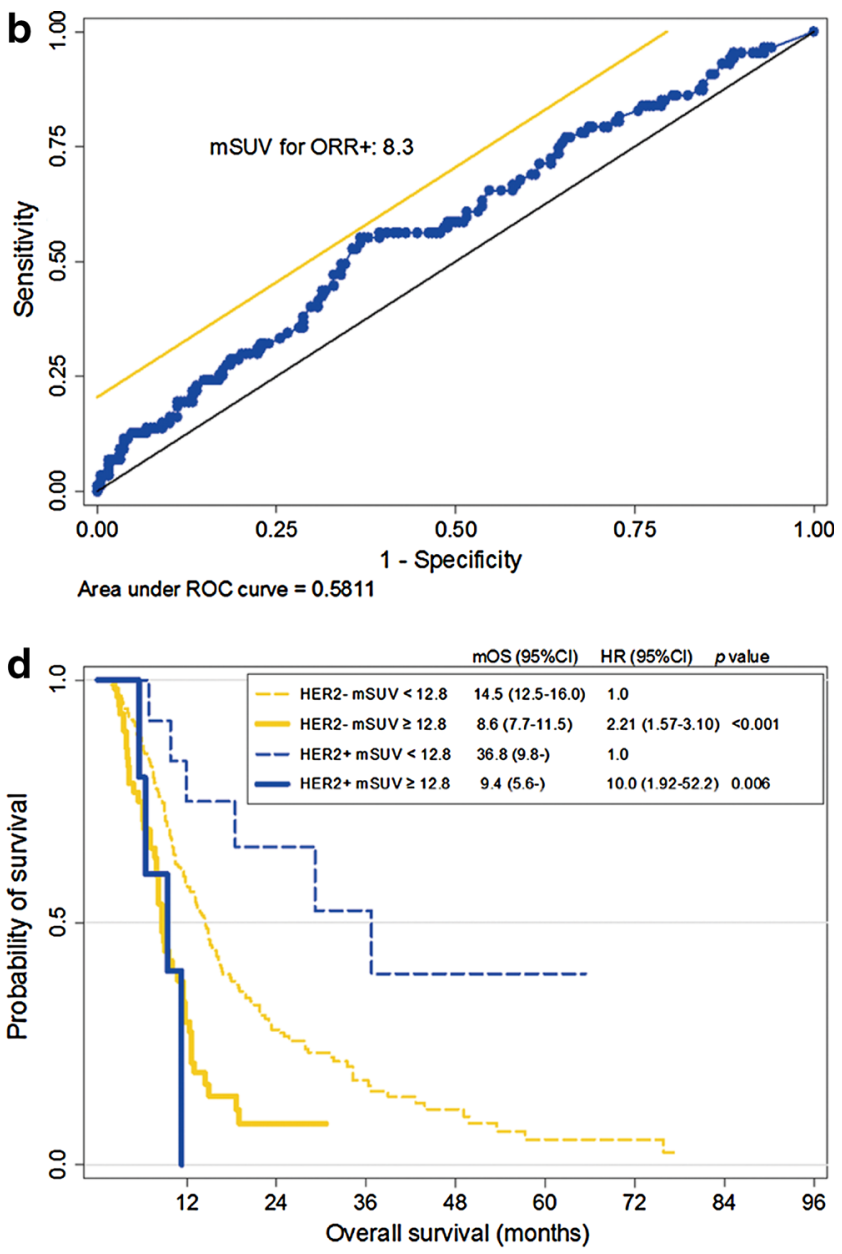

response rate (b) were determined by receiver-operating characteristic curves. Kaplan-Meier curves for OS according to an mSUV cutoff value of 12.8 (c) and according to mSUV and HER2 status (d)

Moreover, a certain report showed that higher HER2 gene amplification was associated with good prognosis [19]. Even though most studies were based on curatively resected cases, the prognostic role of HER2 in AGC is still under debate.

The general characteristics of HER2+ AGC shown in this study were in concordance with previous results [20]. HER2 + was predominantly observed with older patients, males, adenocarcinoma, and intestinal-type pathology. Metastasis to lymph nodes and liver was more prevalent in HER2+ AGC. Maximal SUV and SUVs of stomach and lymph nodes were higher in HER2+ AGC than HER2AGC. Moreover, SUVs of stomach and lymph nodes were higher in HER2+ AGC than HER2- AGC, with a statistical significance.

As far as we know, this is the first report that compares levels and distributions of SUV in whole metastatic lesions according to HER2 status in AGC. Since HER2 is believed to play a role in cell proliferation and migration [8] and 
Table 2 Univariate and multivariate Cox analysis of overall survival

\begin{tabular}{|c|c|c|c|c|c|c|}
\hline & \multirow[t]{2}{*}{$n$} & \multirow[t]{2}{*}{$\mathrm{mOS}$} & \multicolumn{2}{|l|}{ Univariate of OS } & \multicolumn{2}{|l|}{ Multivariate of OS } \\
\hline & & & $\mathrm{HR}(95 \% \mathrm{CI})$ & $p$ & $\mathrm{HR}(95 \% \mathrm{CI})$ & $p$ \\
\hline \multicolumn{7}{|l|}{ mSUV } \\
\hline$<12.8$ & 234 & 14.8 & 1.0 & & 1.0 & \\
\hline$\geq 12.8$ & 62 & 8.6 & $2.38(1.72-3.30)$ & $<0.001$ & $2.34(1.65-3.33)$ & $<0.001$ \\
\hline \multicolumn{7}{|l|}{ Age } \\
\hline$<60$ & 336 & 11.8 & 1.0 & & 1.0 & \\
\hline$\geq 60$ & 362 & 13.1 & $0.84(0.71-1.00)$ & 0.047 & $0.88(0.66-1.18)$ & 0.390 \\
\hline \multicolumn{7}{|l|}{ Sex } \\
\hline Male & 482 & 12.6 & 1.0 & & - & \\
\hline Female & 216 & 12.1 & $1.05(0.87-1.26)$ & 0.595 & - & - \\
\hline \multicolumn{7}{|l|}{ ECOG } \\
\hline $0-1$ & 579 & 13.0 & 1.0 & & 1.0 & \\
\hline $2-3$ & 59 & 6.5 & $2.68(1.99-3.60)$ & $<0.001$ & $1.88(1.20-2.93)$ & 0.006 \\
\hline \multicolumn{7}{|c|}{ Palliative setting } \\
\hline Recur & 214 & 13.1 & 1.0 & & 1.0 & \\
\hline Mets & 484 & 12.1 & $1.22(1.01-1.48)$ & 0.036 & $0.98(0.72-1.32)$ & 0.875 \\
\hline \multicolumn{7}{|c|}{ Tumor location } \\
\hline Other & 639 & 12.5 & 1.0 & & - & \\
\hline GEJ & 59 & 12.1 & $0.90(0.66-1.24)$ & 0.535 & - & - \\
\hline \multicolumn{7}{|c|}{ Pathology: SRC } \\
\hline No & 500 & 13.2 & 1.0 & & 1.0 & \\
\hline Yes & 198 & 10.5 & $1.35(1.12-1.64)$ & 0.002 & $1.25(0.90-1.75)$ & 0.187 \\
\hline \multicolumn{7}{|l|}{ HER2 } \\
\hline- & 641 & 12.5 & 1.0 & & - & \\
\hline+ & 57 & 11.9 & $0.94(0.70-1.26)$ & 0.688 & - & - \\
\hline \multicolumn{7}{|c|}{ Lymph node metastasis } \\
\hline No & 376 & 13.0 & 1.0 & & - & \\
\hline Yes & 322 & 12.1 & $1.01(0.85-1.21)$ & 0.870 & - & - \\
\hline \multicolumn{7}{|c|}{ Peritoneal seeding } \\
\hline No & 274 & 13.4 & 1.0 & & 1.0 & \\
\hline Yes & 424 & 11.7 & $1.32(1.10-1.58)$ & 0.002 & $1.61(1.18-2.18)$ & 0.003 \\
\hline \multicolumn{7}{|c|}{ Liver metastasis } \\
\hline No & 540 & 13.1 & 1.0 & & 1.0 & \\
\hline Yes & 158 & 11.6 & $1.31(1.08-1.61)$ & 0.007 & $1.48(1.04-2.10)$ & $\mathbf{0 . 0 2 7}$ \\
\hline
\end{tabular}

Bold values indicate statistically significant correlations with $p$ values less than 0.05

CI confidence interval, ECOG Eastern Cooperative Oncology Group performance status, GEJ gastroesophageal junction, $H E R 2$ human epidermal growth factor receptor 2, $H R$ hazard ratio, $m O S$ median overall survival, $m S U V$ maximal standardized uptake value, $S R C$ signet ring cell
SUV correlates with tumor cell metabolism [21], HER2+ AGC would be a more hypermetabolic tumor compared with HER2- AGC on the basis of this analysis. Several factors may influence these results. First of all, the proportion of signet ring cell pathology with an SUV lower than that of adenocarcinoma [22-25] was significantly low in HER2+ AGC. However, when we performed subgroup analysis of the mSUV difference based on pathologic type, the mean mSUV of patients with the signet ring cell feature was also significantly increased in HER2+ AGC compared to HER2- AGC (data not shown). Second, the proportion of patients with initially metastatic stage IV, which generally had more tumor burden than recurrent cases, was slightly higher in HER2 + AGC in our cohort, but it did not differ significantly (HER2-: $69.3 \%$ vs. HER2+: $72.9 \%$ ). Moreover, the rate of availability of ${ }^{18} \mathrm{~F}$ FDG-PET data was well balanced according to HER2 status. Third, other markers associated with HER2 would also influence the hypermetabolism of the HER2+ tumor. Recently, The Cancer Genome Atlas (TCGA) genomic analysis of gastric cancer reported recurrent co-amplifications of the receptor tyrosine kinase family, such as EGFR, FGFR2, VEGFR2, 
and $M E T$, as well as cell cycle regulators, such as CCNE1, $C D K 6$, and $C C N D 1$ along with $H E R 2$, especially in the chromosomal-instable (CIN) subtype of gastric cancer [26, 27]. Hence, the hypermetabolism of HER $2+$ tumor might be derived from HER2 itself or other markers including the receptor tyrosine kinase family and cell cycle regulators associated with HER2. The clarification of tumor metabolism by those factors should be further investigated. Taken together, we interpret our current data macroscopically as showing a difference in tumor metabolism based on HER2 status, although it needs to be further validated.

In our cohort, although mSUV was positively correlated with HER2 protein expression seen by IHC and the HER2/ CEP17 ratio was increased with a higher IHC positivity (Supplementary Table 1), the HER2/CEP17 ratio and HER2 GCN were not related to mSUV. Therefore, HER2 protein expression rather than HER2 gene amplification may play a more crucial role in representing influences on tumor metabolism.

Irrespective of HER2 status, tumor hypermetabolism negatively influenced OS. It also suggests that other factors besides HER2 influence hypermetabolism of gastric cancer. For example, tumor metabolism is consistently influenced by the mutation status of the MYC, TP53, and LKB1AMPK-PI3 K pathway [28]. Therefore, cancer metabolism seems far more pleiotropic than it is expected to be.

Tumor hypermetabolism in gastric cancer was correlated with a higher response rate to conventional cytotoxic chemotherapy in our study. Recent data showed that the tumor turnover rate is dependent on glucose metabolism [29]. Therefore, cytotoxic chemotherapy could be more effective in tumors with hypermetabolism, but tumor progression would be more rapid because of its aggressiveness.

Surprisingly, although HER2+ AGC is a hypermetabolic tumor and generally hypermetabolism is associated with poor prognosis, OS of HER2+ AGC was not worse than that of HER2- AGC in the current study. This effect would be explained by exclusion of HER2+ AGC patients with higher mSUV when performing survival analysis, because of trastuzumab exposure. Uneven trastuzumab treatment to HER2 + AGC according to the level of mSUV may be an incidental finding, since the decision for trastuzumab treatment was not based on the mSUV in clinical practice. Moreover, the number of patients with AGC without exposure to HER2-targeting agents is relatively small, since trastuzumab treatment in HER2+ AGC is the standard of care after the ToGA study [6], where the statistical significance could be underpowered.

In conclusion, tumor metabolism is higher in HER2+ AGC, and this metabolic activity adversely influences OS. However, HER2 itself is not a prognostic factor in AGC patients who receive cytotoxic chemotherapy excluding
HER2-targeting agents. Further evaluation should be focused on measuring tumor metabolism with other methods besides ${ }^{18} \mathrm{~F}$ FDG-PET to understand the biologic roles of HER2 in AGC.

Acknowledgments This research was supported by the Basic Science Research Program through the National Research Foundation of Korea (NRF) funded by the Ministry of Education, Science and Technology (grant no. 2013R1A1A2008705).

\section{References}

1. Lordick F, Lorenzen S, Yamada Y, Ilson D. Optimal chemotherapy for advanced gastric cancer: is there a global consensus? Gastric Cancer. 2014;17:213-25.

2. Kang JH, Lee SI, Lim do H, Park KW, Oh SY, Kwon HC, et al. Salvage chemotherapy for pretreated gastric cancer: a randomized phase III trial comparing chemotherapy plus best supportive care with best supportive care alone. J Clin Oncol. 2012;30:1513-8.

3. Ford HE, Marshall A, Bridgewater JA, Janowitz T, Coxon FY, Wadsley J, et al. Docetaxel versus active symptom control for refractory oesophagogastric adenocarcinoma (COUGAR-02): an open-label, phase 3 randomised controlled trial. Lancet Oncol. 2014;15:78-86.

4. Deng N, Goh LK, Wang H, Das K, Tao J, Tan IB, et al. A comprehensive survey of genomic alterations in gastric cancer reveals systematic patterns of molecular exclusivity and co-occurrence among distinct therapeutic targets. Gut. 2012;61:673-84.

5. Dulak AM, Schumacher SE, van Lieshout J, Imamura Y, Fox C, Shim B, et al. Gastrointestinal adenocarcinomas of the esophagus, stomach, and colon exhibit distinct patterns of genome instability and oncogenesis. Cancer Res. 2012;72:4383-93.

6. Bang YJ, Van Cutsem E, Feyereislova A, Chung HC, Shen L, Sawaki A, et al. Trastuzumab in combination with chemotherapy versus chemotherapy alone for treatment of HER2-positive advanced gastric or gastro-oesophageal junction cancer (ToGA): a phase 3, open-label, randomised controlled trial. Lancet. 2010;376:687-97.

7. Slamon DJ, Clark GM, Wong SG, Levin WJ, Ullrich A, McGuire WL. Human breast cancer: correlation of relapse and survival with amplification of the HER-2/neu oncogene. Science. 1987;235:177-82.

8. Gravalos C, Jimeno A. HER2 in gastric cancer: a new prognostic factor and a novel therapeutic target. Ann Oncol. 2008;19:1523-9.

9. Tanner M, Hollmen M, Junttila TT, Kapanen AI, Tommola S, Soini Y, et al. Amplification of HER-2 in gastric carcinoma: association with Topoisomerase IIalpha gene amplification, intestinal type, poor prognosis and sensitivity to trastuzumab. Ann Oncol. 2005;16:273-8.

10. Kim JW, Im SA, Kim M, Cha Y, Lee KH, Keam B, et al. The prognostic significance of HER2 positivity for advanced gastric cancer patients undergoing first-line modified FOLFOX-6 regimen. Anticancer Res. 2012;32:1547-53.

11. Qiu MZ, Li Q, Wang ZQ, Liu TS, Liu Q, Wei XL, et al. HER2positive patients receiving trastuzumab treatment have a comparable prognosis with HER2-negative advanced gastric cancer patients: a prospective cohort observation. Int $\mathrm{J}$ Cancer. 2014;134:2468-77. 
12. Grabsch H, Sivakumar S, Gray S, Gabbert HE, Muller W. HER2 expression in gastric cancer: rare, heterogeneous and of no prognostic value-conclusions from 924 cases of two independent series. Cell Oncol. 2010;32:57-65.

13. Janjigian YY, Werner D, Pauligk C, Steinmetz K, Kelsen DP, Jager E, et al. Prognosis of metastatic gastric and gastroesophageal junction cancer by HER2 status: a European and USA International collaborative analysis. Ann Oncol. 2012;23:2656-62.

14. Shitara K, Yatabe Y, Matsuo K, Sugano M, Kondo C, Takahari $\mathrm{D}$, et al. Prognosis of patients with advanced gastric cancer by HER2 status and trastuzumab treatment. Gastric Cancer. 2013;16:261-7.

15. Fisher SB, Fisher KE, Squires MH 3rd, Patel SH, Kooby DA, ElRayes BF, et al. HER2 in resected gastric cancer: is there prognostic value? J Surg Oncol. 2014;109:61-6.

16. Choi H, Paeng JC, Kim DW, Lee JK, Park CM, Kang KW, et al. Metabolic and metastatic characteristics of ALK-rearranged lung adenocarcinoma on FDG PET/CT. Lung Cancer. 2013;79:242-7.

17. Eisenhauer EA, Therasse P, Bogaerts J, Schwartz LH, Sargent D, Ford R, et al. New response evaluation criteria in solid tumours: revised RECIST guideline (version 1.1). Eur J Cancer. 2009;45:228-47.

18. Youden WJ. Index for rating diagnostic tests. Cancer. 1950;3:32-5.

19. Gomez-Martin C, Garralda E, Echarri MJ, Ballesteros A, Arcediano A, Rodriguez-Peralto JL, et al. HER2/neu testing for antiHER2-based therapies in patients with unresectable and/or metastatic gastric cancer. J Clin Pathol. 2012;65:751-7.

20. Chua TC, Merrett ND. Clinicopathologic factors associated with HER2-positive gastric cancer and its impact on survival outcomes-a systematic review. Int J Cancer. 2012;130:2845-56.
21. Zhu A, Lee D, Shim H. Metabolic positron emission tomography imaging in cancer detection and therapy response. Semin Oncol. 2011;38:55-69.

22. Alakus H, Batur M, Schmidt M, Drebber U, Baldus SE, Vallbohmer D, et al. Variable 18F-fluorodeoxyglucose uptake in gastric cancer is associated with different levels of GLUT-1 expression. Nucl Med Commun. 2010;31:532-8.

23. Kole AC, Plukker JT, Nieweg OE, Vaalburg W. Positron emission tomography for staging of oesophageal and gastroesophageal malignancy. Br J Cancer. 1998;78:521-7.

24. Sim SH, Kim YJ, Oh DY, Lee SH, Kim DW, Kang WJ, et al. The role of PET/CT in detection of gastric cancer recurrence. BMC Cancer. 2009;9:73.

25. Yoshioka T, Yamaguchi K, Kubota K, Saginoya T, Yamazaki T, Ido T, et al. Evaluation of 18F-FDG PET in patients with advanced, metastatic, or recurrent gastric cancer. J Nucl Med. 2003;44:690-9.

26. Cancer Genome Atlas Research Network. Comprehensive molecular characterization of gastric adenocarcinoma. Nature. 2014;513:202-9.

27. Kim J, Fox C, Peng S, Pusung M, Pectasides E, Matthee E, et al. Preexisting oncogenic events impact trastuzumab sensitivity in ERBB2-amplified gastroesophageal adenocarcinoma. J Clin Invest. 2014;124:5145-58.

28. Boroughs LK, DeBerardinis RJ. Metabolic pathways promoting cancer cell survival and growth. Nat Cell Biol. 2015;17:351-9.

29. Bensinger SJ, Christofk HR. New aspects of the Warburg effect in cancer cell biology. Semin Cell Dev Biol. 2012;23:352-61. 